OPEN ACCESS

Edited by:

Cornelis F. M. Sier,

Leiden University, Netherlands

Reviewed by:

Vladimir Cuk

Clinical Hospital Center Zvezdara,

Serbia

Ali Coskun,

Izmir Bozyaka Eğitim ve Araştırma

Hastanesi, Turkey

*Correspondence:

Chun-Xi Wang

bjwangcx@126.com

Jian-Hai Long

jianhai8561729@sina.com

Specialty section:

This article was submitted to Gastrointestinal Cancers,

a section of the journa

Frontiers in Oncology

Received: 13 February 2019

Accepted: 11 April 2019

Published: 03 May 2019

Citation:

Yang Z-L, Zhu M-H, Han X-J,

Liu Q-W, Long J-H and Wang C-X (2019) Modified American Joint

Committee on Cancer

Tumor-Node-Metastasis Staging

System Based on the Node Ratio Can

Further Improve the Capacity of

Prognosis Assessment for Gastric Cancer Patients. Front. Oncol. 9:329.

doi: 10.3389/fonc.2019.00329

\section{Modified American Joint Committee on Cancer Tumor-Node-Metastasis Staging System Based on the Node Ratio Can Further Improve the Capacity of Prognosis Assessment for Gastric Cancer Patients}

\author{
Ze-Long Yang ${ }^{1,2}$, Ming-Hua Zhu ${ }^{1}$, Xiu-Jing Han ${ }^{3}$, Qiang-Wei Liu ${ }^{4}$, Jian-Hai Long ${ }^{5 *}$ and \\ Chun-Xi Wang ${ }^{1,2 *}$
}

\begin{abstract}
'Department of General Surgery, Chinese People's Liberation Army General Hospital, Beijing, China, ${ }^{2}$ Department of General Surgery, Hainan Hospital of PLA General Hospital, Sanya, China, ${ }^{3}$ Clinical Laboratory, The First Affiliated Hospital of

Guangzhou Medical University, Guangzhou, China, ${ }^{4}$ Anesthesiology and Operation Center, Chinese People's Liberation Army General Hospital, Beijing, China, ${ }^{5}$ Department of General Surgery, People's Liberation Army No. 520 Hospital, Mianyang, China
\end{abstract}

Background and Objectives: Our aim was to investigate whether the modified American Joint Committee on Cancer (AJCC) tumor-node-metastasis (TNM) staging system based on the node ratio can further improve the capacity of prognosis assessment for gastric cancer (GC) patients regardless of the number of lymph nodes examined (eLNs).

Methods: A total of 17,187 GC patients in the Surveillance, Epidemiology, and End Results (SEER) database were included. On the basis of a training set of 7,660 GC patients, we built the tumor-node ratio-metastasis (TNrM) staging system, which was then externally validated with a validation set of 9,527 GC patients.

Results: For the training set, the C-index value of the TNrM staging system was significantly higher than that of the AJCC 8th TNM staging system to predict survival for GC patients (C-index: 0.688 vs. 0.671, $P<0.001$ ). Moreover, the $\mathrm{C}$-index value of the TNrM staging system was significantly higher than that of the 8th TNM staging system to predict survival for GC patients with $\leq 15 \mathrm{eLNs}$ (C-index: 0.682 vs. $0.673, P<0.001$ ), as well as for GC patients with $>15$ eLNs (C-index: 0.700 vs. $0.694, P<0.001$ ). Similar results were found in the validation set.

Conclusions: The TNrM staging system predicted survival more accurately and discriminatively than the AJCC 8th TNM staging system for GC patients regardless of the number of eLNs.

Keywords: gastric adenocarcinoma, tumor-node-metastasis, tumor-node ratio-metastasis, lymph nodes examined, survival 


\section{INTRODUCTION}

Gastric cancer (GC) is the second leading cause of cancerrelated death and the fifth most common cancer worldwide (1). An accurate and discriminative staging system is necessary for doctors to assess the prognosis of patients and to make appropriate medical decisions. The most commonly used staging system for GC is the International Union Against Cancer (UICC) or American Joint Committee on Cancer (AJCC) tumor-nodemetastasis (TNM) staging system (2). The TNM staging system continues to base the pathologic $\mathrm{N}$ category $(\mathrm{pN})$ definitions on the absolute number of regional metastatic lymph nodes (3). However, the $\mathrm{pN}$ stage could have a stage migration because the number of metastatic lymph nodes can change according to the number of lymph nodes examined (eLNs). Meanwhile, the number of eLNs is affected by various factors, including the extent of lymphadenectomy, types of gastrectomy, characteristics of tumor, and skills of operators, thus many factors could influence the prognostic performance of the $\mathrm{N}$ staging system. Considering this, previous studies have proposed modifications to the TNM staging system by including the positive lymph node ratio (LNR). Node ratio $(\mathrm{Nr})$ is defined as the number of metastatic lymph nodes divided by the number of eLNs (4). Several studies further confirmed that the tumor-node ratiometastasis (TNrM) staging system was more appropriate than the AJCC 7th TNM staging system, and the main interpretation for that involved stage migration (5-11). Recently, the AJCC 8th TNM staging system has made several modifications to the 7th TNM staging system, including inclusion of $\mathrm{pN} 3 \mathrm{~b}$ into the staging system and modifications of existing staging subgroups (3). Subsequently, populations from both the West and the East have validated the AJCC 8th TNM staging system's superiority over the AJCC 7th TNM staging system for patients with adequate eLNs (12-15). However, the validity and superiority of the AJCC 8th TNM staging system compared with the TNrM staging system remain unknown. On the other hand, patients with adequate eLNs will have a slight risk of stage migration (16). Therefore, it should be questioned whether the TNrM staging system was superior than the AJCC 8th TNM staging system when predicting survival for patients with adequate eLNs.

In the light of these considerations, the aims of our present study were the following: (1) to modify the AJCC 8th TNM staging system based on the $\mathrm{Nr}$; (2) to investigate whether the TNrM staging system was superior to the AJCC 8th TNM staging system to predict survival for GC patients regardless of the number of eLNs.

\section{MATERIALS AND METHODS}

\section{Patients}

In this retrospective analysis, patients who underwent gastrectomy in the Surveillance, Epidemiology, and End Results (SEER) database with at least 18 years of age with histology-confirmed GC (ICD-O-3: M-8140/3, M-8142/3 to M$8145 / 3$, M-8210/3, M-8211/3, M-8255/3, M-8260/3 to M-8263/3, M-8310/3, M-8323/3) were included. The detailed included process for GC patients is shown in Supplemental Figure 1.
Finally, a total of 17,187 GC patients were included for this study. Further, these patients were classified into training set and validation set based on the SEER registries following our previous study (17). The median follow-up time for training and validation sets was 162 and 100 months, respectively.

\section{Statistical Methods}

To build the $\mathrm{Nr}$ staging system, we determined the cutoff values of the LNR as being where the $\mathrm{Nr}$ subgroups showed the 5-year overall survival (OS) that was the most similar. A unique stratification was developed for node-negative GC patients because of the fact that the LNR value for these patients are always the same, which will not be influenced by the number of eLNs. The optimal number of eLNs that determined the most significantly survival difference for node-negative GC patients was 9 (Supplemental Figure 2) using the X-tile software (http:// www.tissuearray.org) (18). Then, $5 \mathrm{Nr}$ stages were established by combining the neighborhood survival curves according to the 5year OS (Nr0: LNR $=0$, eLNs $>$ 9; Nr1: LNR $=0$, eLNs $\leq$ 9, or $0<\mathrm{LNR} \leq 0.1 ; \mathrm{Nr} 2: 0.1<\mathrm{LNR} \leq 0.3$; Nr3a: $0.3<\mathrm{LNR}$ $\leq 0.7$; and Nr3b: LNR $>0.7$; Figure 1). In order not to further complicate an already complex staging system, the TNrM staging system was established by combining the same $\mathrm{pT}$ and $\mathrm{pM}$ from the AJCC 8th TNM staging system with the Nr instead of the pN (Supplemental Table 1).

The OS probabilities were calculated by the Kaplan-Meier method, and the different OS probabilities between groups were examined by log-rank test. The likelihood ratio test within a

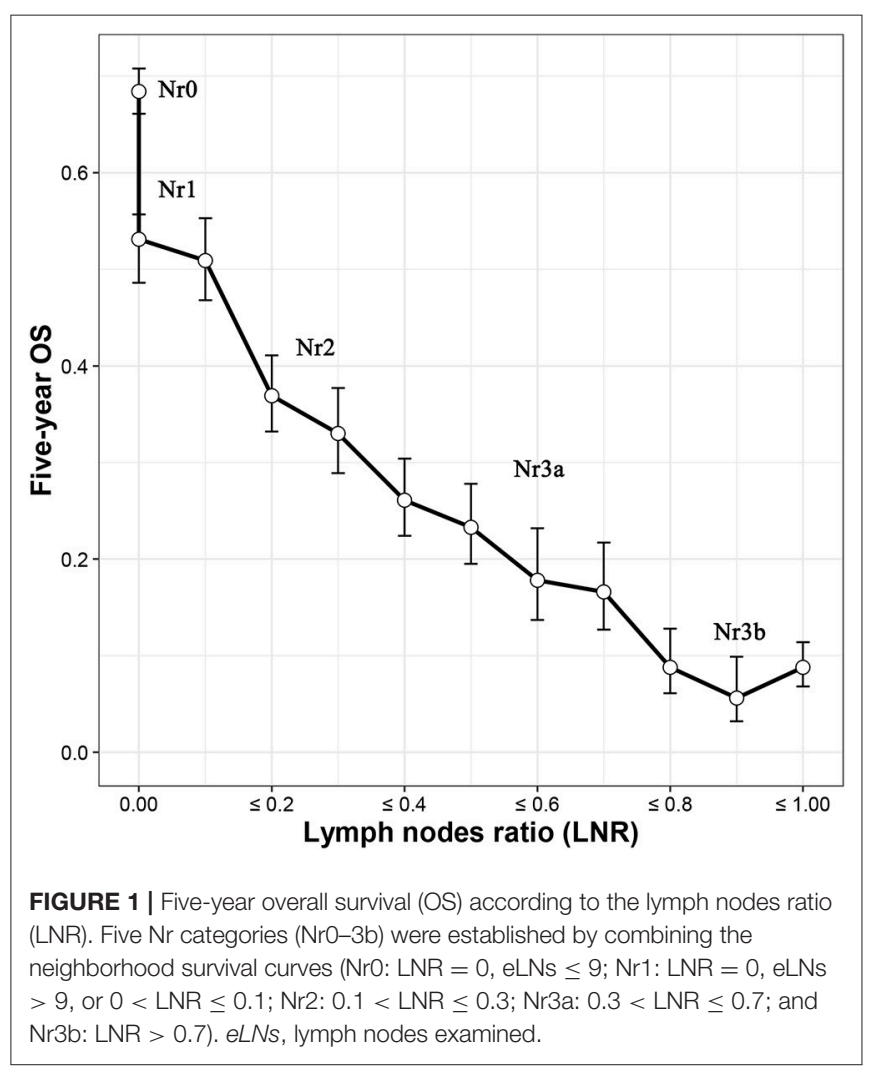


Cox regression model was used to assess the homogeneity of the staging system. Harrell's concordance index (C-index) was used to assess the relative discriminatory ability of the staging system, and a value closer to 1 means a higher predictive ability. Furthermore, the Akaike information criterion (AIC) within a Cox regression model was also used to measure the discriminatory ability of the staging system, and a smaller AIC value means a more reasonable model for prognosis (19). All statistical analyses were performed using R-3.4 software (http://www.r-project.org). $P<0.05$ (two-sided) was considered statistically significant.

\section{RESULTS}

\section{Patient Demographics and Tumor Characteristics}

The included process for GC patients is shown in Supplemental Figure 1. Finally, 17,187 GC patients with complete data for staging were identified. Of these, 7,660 patients were classified into the training set and 9,527 patients were classified into the validation set. The median number of eLNs for the training set was 12 (interquartile range [IQR],7-20), and for the validation set it was 13 (IQR, 7-20). The median OS time for training set was 38 (IQR,13-129) months and validation set was 42 (IQR, 13-149) months. Detailed characteristics of GC patients for the training and validation sets are summarized in Supplemental Table 2.

\section{Prognostic Performance of Different Staging Systems in the Training Set}

For the training set, each $\mathrm{pN}$ category ( $\mathrm{pN} 0-3 \mathrm{~b})$ was stratified into $\mathrm{Nr}$ subgroups. Each $\mathrm{pN}$ category ( $\mathrm{pN} 0-3 \mathrm{~b})$ was found to contain subgroups of patients with significantly heterogeneous OS (all $P<0.001$; Table 1). Similarly, each $\mathrm{Nr}$ category ( $\mathrm{Nr} 0-$ $3 \mathrm{~b}$ ) was stratified into $\mathrm{pN}$ subgroups; only the $\mathrm{Nr} 3 \mathrm{~b}$ category was found to contain subgroups of patients with significantly heterogeneous OS $(P<0.001$; Table 1). Moreover, the OS of each $\mathrm{pN}$ category ( $\mathrm{pN} 0-3 \mathrm{~b}$ ) for patients with $>15$ eLNs was significantly better than that of patients with $\leq 15$ eLNs (all $P$ $<0.001$; Table 2). Conversely, the significantly different OS was found in the $\mathrm{Nr} 0, \mathrm{Nr} 2$, and $\mathrm{Nr} 3 \mathrm{~b}$ categories for patients with $>15$ eLNs and patients with $\leq 15$ eLNs (Nr0: $P=0.028$; Nr2: $P=$ 0.049; Nr3b: $P=0.008$; Table 2), and the OS of Nr1and Nr3a categories for patients $>15$ eLNs was similar to that of patients with $\leq 15$ eLNs (all $P>0.05$; Table 2). These results indicated that compared with the $\mathrm{pN}$ categories, $\mathrm{Nr}$ categories represent

TABLE 1 | Five-year overall survival by the AJCC 8th N staging system and the Nr staging system for the training set.

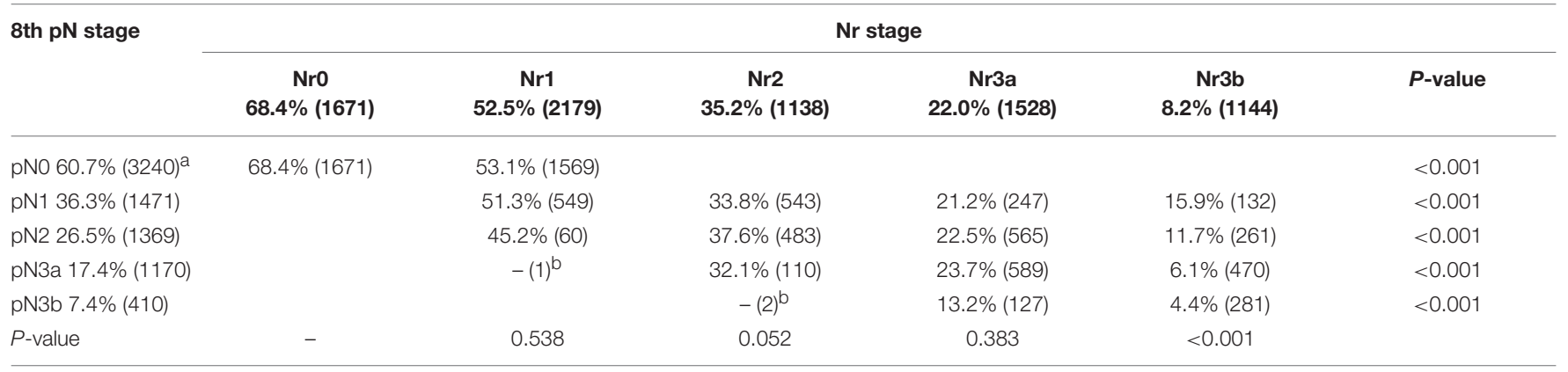

AJCC, American Joint Committee on Cancer.

a Five-year overall survival was calculated by Kaplan-Meier method, followed the number of patients.

${ }^{b}$ Five-year overall survival was not calculated for this subgroup because of limited number of patients.

TABLE 2 | Five-year OS by the AJCC 8th N staging system and the Nr staging system according to the number of eLNs.

\begin{tabular}{|c|c|c|c|c|c|c|c|}
\hline 8th pN stage & No. of eLNs & Five-year OS & $P$-value & Nr stage & No. of eLNs & Five-year OS & $P$-value \\
\hline \multirow[t]{2}{*}{ pNO $60.7 \%(3240)^{a}$} & eLNs > 15 & $70.5 \%(1000)$ & reference & NrO 68.4\% (1671) & eLNs > 15 & $70.5 \%(1000)$ & reference \\
\hline & eLNs $\leq 15$ & $56.7 \%(2240)$ & $<0.001$ & & eLNs $\leq 15$ & $65.5 \%(671)$ & 0.028 \\
\hline \multirow[t]{2}{*}{ pN1 36.3\% (1471) } & eLNs > 15 & $53.7 \%(409)$ & reference & Nr1 52.5\% (2179) & eLNs > 15 & $54.5 \%(420)$ & reference \\
\hline & $\mathrm{eLNs} \leq 15$ & $30.0 \%(1062)$ & $<0.001$ & & $\mathrm{eLNs} \leq 15$ & $52.1 \%(1759)$ & 0.194 \\
\hline \multirow[t]{2}{*}{ pN2 26.5\% (1369) } & eLNs > 15 & $38.8 \%(452)$ & reference & Nr2 35.2\% (1138) & eLNs > 15 & $37.9 \%(505)$ & reference \\
\hline & $\mathrm{eLNs} \leq 15$ & $20.9 \%(917)$ & $<0.001$ & & $\mathrm{eLNs} \leq 15$ & $33.3 \%(633)$ & 0.049 \\
\hline \multirow[t]{2}{*}{ pN3a 17.4\% (1170) } & eLNs > 15 & $23.9 \%(623)$ & reference & Nr3a 22.0\% (1528) & eLNs > 15 & $22.4 \%(610)$ & reference \\
\hline & $\mathrm{eLNs} \leq 15$ & $10.2 \%(547)$ & $<0.001$ & & $\mathrm{eLNs} \leq 15$ & $21.7 \%(918)$ & 0.510 \\
\hline \multirow[t]{2}{*}{ pN3b 7.4\% (410) } & eLNs > 15 & $7.4 \%(410)$ & - & Nr3b 8.2\% (1144) & eLNs > 15 & $5.0 \%(359)$ & reference \\
\hline & & & & & $\mathrm{eLNs} \leq 15$ & $9.6 \%(785)$ & 0.008 \\
\hline
\end{tabular}

AJCC, American Joint Committee on Cancer; eLNs, lymph nodes examined; OS, overall survival.

a Five-year OS was calculated by Kaplan-Meier method, followed the number of patients. 
patients with more homogeneous OS. The $\mathrm{Nr}$ staging system had a higher likelihood ratio $\chi^{2}$ value and thus also represented better homogeneity (Table 3 ).

The survival curves for the training set are shown in Figure 2. The C-index value of the $\mathrm{Nr}$ staging system was significantly higher than that of the $\mathrm{pN}$ staging system to predict survival for GC patients (C-index: 0.666 vs. $0.641, P<0.001$; Table 3). The TNrM staging system was constructed on the basis of the same pT and pM with the new $\mathrm{Nr}$ (Supplemental Table 1). Furthermore, we compared the prognostic performance for these two staging systems. The TNrM staging system performed better than the AJCC 8th TNM staging system to predict survival for GC patients (C-index: 0.688 vs. $0.671, P<0.001$; Table 3 ). In order to evaluate the influence of the number of eLNs on the prognostic performance of these two staging systems, we classified the GC patients into 2 groups according to the number of eLNs: group 1 , eLNs $\leq 15$; group 2 , eLNs $>15$. The cutoff point of 15 was chosen because of the fact that 15 lymph nodes was the landmark of eLNs recommended by the National Comprehensive Cancer Network (NCCN) to avoid stage migration (20). For GC patients with $\leq 15$ eLNs, the $\mathrm{Nr}$ staging system and the TNrM staging system performed better than the AJCC staging system to predict survival for these GC patients (C-index: $\mathrm{Nr}$ vs. $\mathrm{pN}, 0.651$ vs. $0.635, P<0.001$; TNrM vs. TNM: 0.682 vs. $0.673, P<0.001$; Table 3). Similarly, for GC patients with $>15$ eLNs, the Nr staging system and the TNrM staging system represented patients with

TABLE 3 | Comparison of the performance of different staging systems.

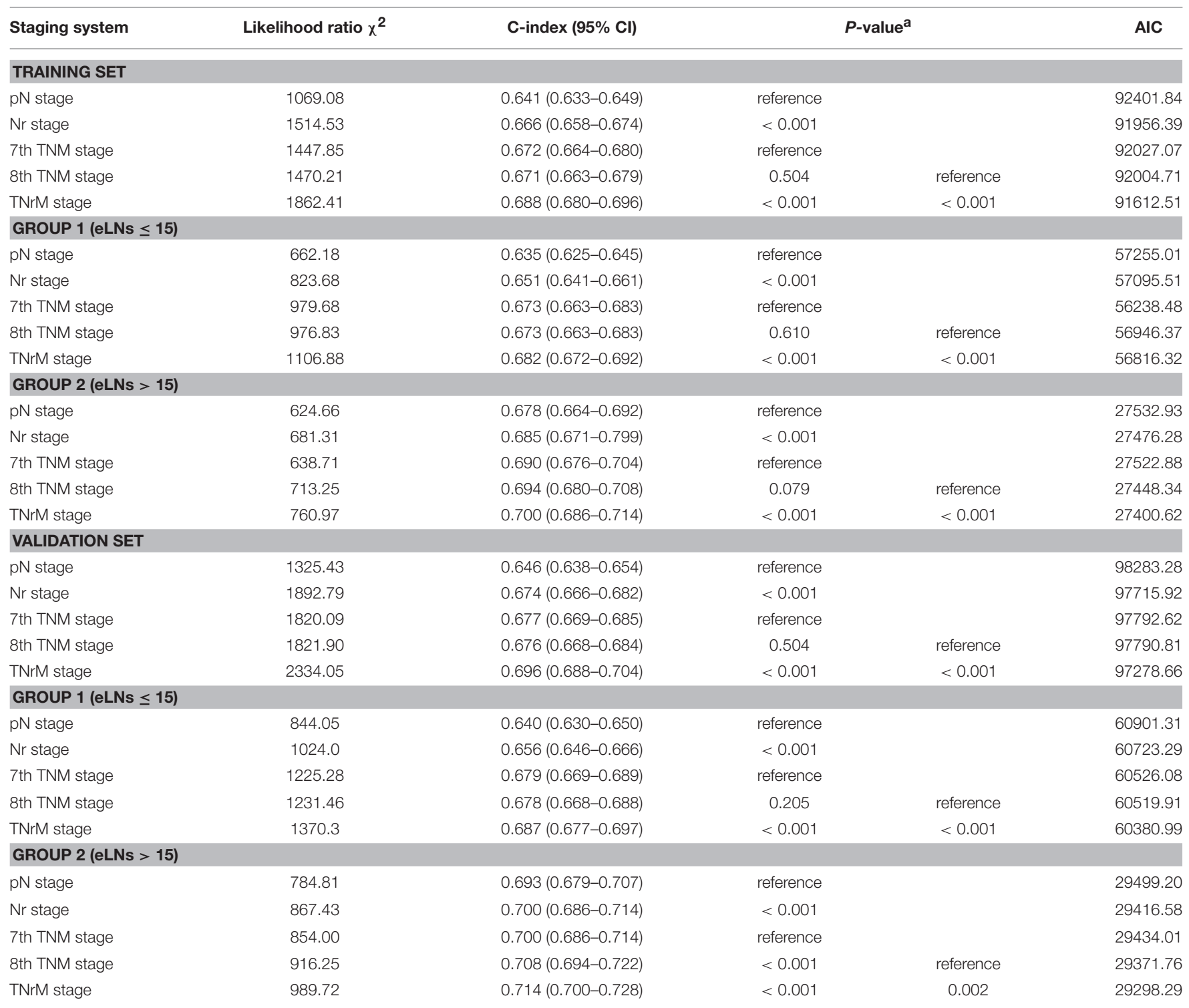

AJCC, American Joint Committee on Cancer; TNM, tumor-node-metastasis; TNrM, tumor-node ratio-metastasis; Cl, confidence interval; AIC, Akaike information criterion; eLNs, lymph nodes examined.

a -value was calculated by $R$ package "compareC." 
A

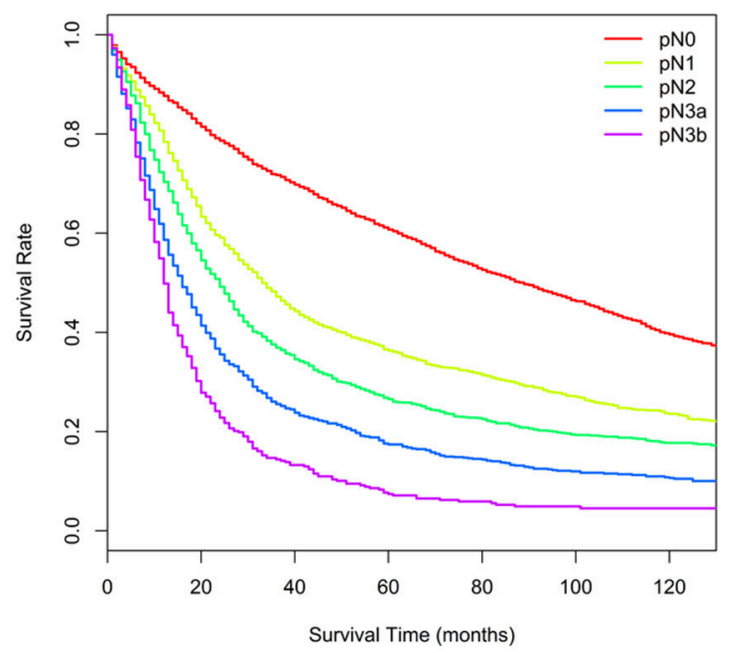

C

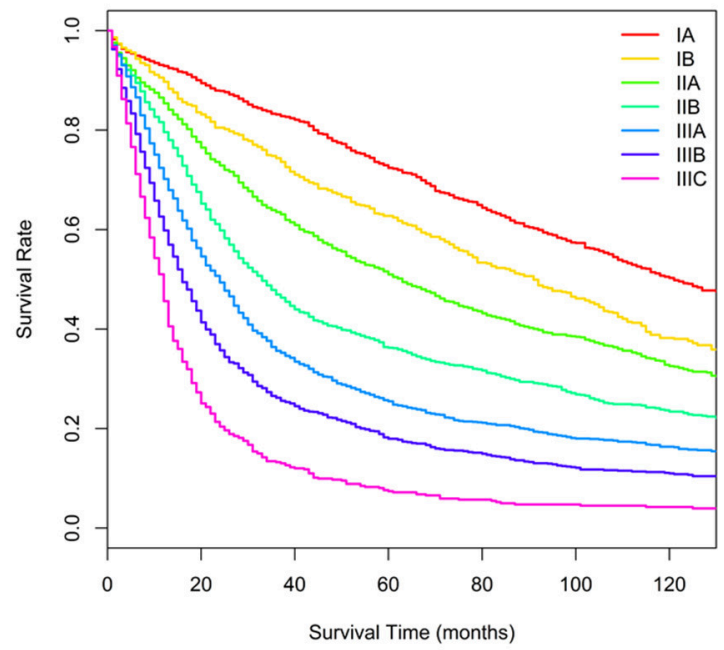

B

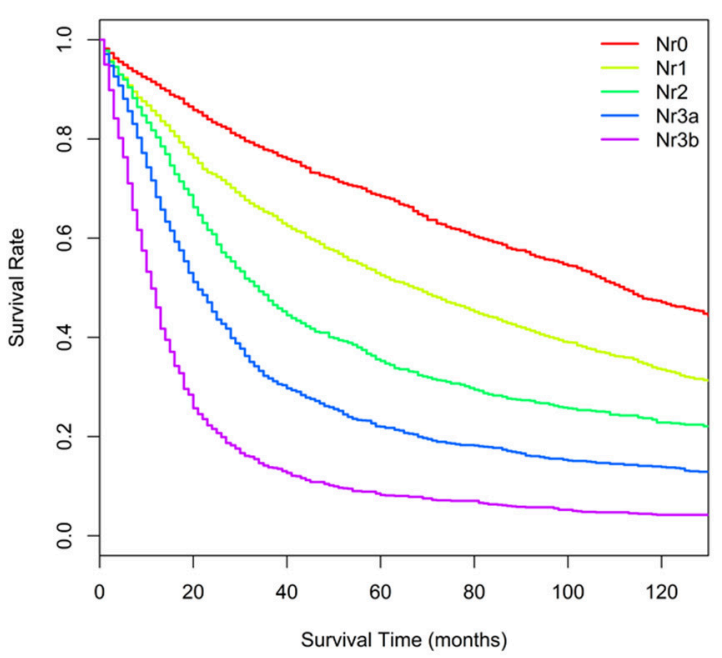

D

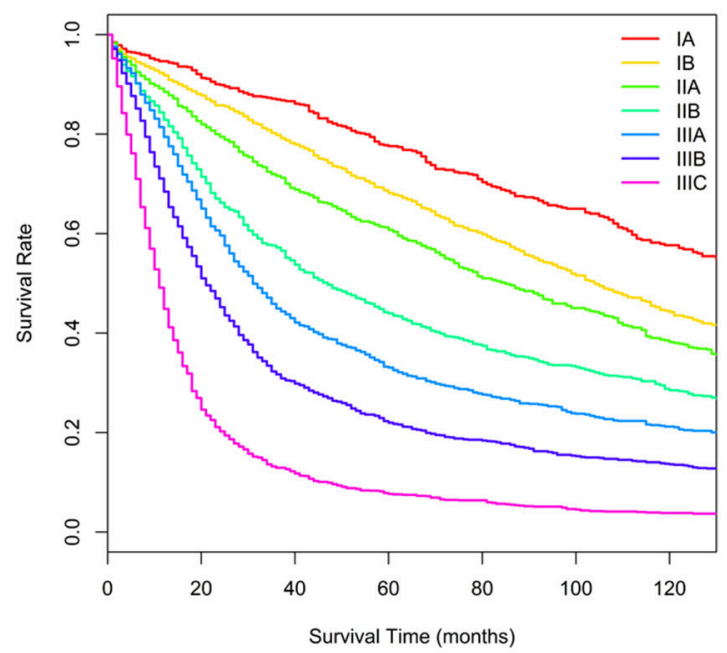

FIGURE 2 | Overall survival (OS) for the training set based on different staging systems. (A) pN staging system. Five-year OS for the pN categories (pNO-3b) was 60.7, 36.3, 26.5, 17.4, and 7.4\%, respectively. (B) Nr staging system. Five-year OS for the Nr categories (NrO-3b) was 68.4, 52.5, 35.3, 22.0, and 8.2\%, respectively. (C) 8th TNM staging system. Five-year OS for the 8th TNM stages (IA-IIIC) was 72.4, 62.8, 51.0, 36.3, 25.5, 18.0, and 7.4\%, respectively. (D) TNrM staging system. Five-year OS for the TNrM stages (IA-IIIC) was 77.6, 68.2, 60.7, 44.0, 33.1, 22.0, and 7.7\%, respectively.

better prognostic identification than the AJCC staging system (Cindex: $\mathrm{Nr}$ vs. $\mathrm{pN}$ : 0.685 vs. $0.678, P<0.001$; TNrM vs. TNM: 0.700 vs. $0.694, P<0.001$; Table 3 ).

\section{Prognostic Performance of Different Staging Systems in the Validation Set}

To validate results from the training set, we conducted similar analyses in the validation set. The survival curves for the validation set are shown in Figure 3. The C-index values of the $\mathrm{Nr}$ and TNrM staging systems were significantly higher than that of the AJCC 8th TNM staging system to predict survival for GC patients (C-index: Nr vs. pN: 0.674 vs. $0.646, P<0.001$; TNrM vs. TNM: 0.696 vs. $0.676, P<0.001$; Table 3 ). Similarly, the C-index values of the $\mathrm{Nr}$ and TNrM staging systems were significantly higher than that of the 8th TNM staging system to predict survival for GC patients with $\leq 15$ eLNs (C-index: $\mathrm{Nr}$ vs. pN: 0.656 vs. $0.640, P<0.001$; TNrM vs. TNM: 0.687 vs. 0.678 , $P<0.001$; Table 3), as well as for GC patients with $>15$ eLNs (C-index: Nr vs. pN: 0.700 vs. $0.693, P<0.001$; TNrM vs. TNM: 0.714 vs. $0.708, P=0.002$; Table 3 ).

\section{DISCUSSION}

An accurate staging system is important for prognostic assessment and tailoring a treatment plan for GC patients. Several studies have validated that the main superiority of 8th TNM 
A

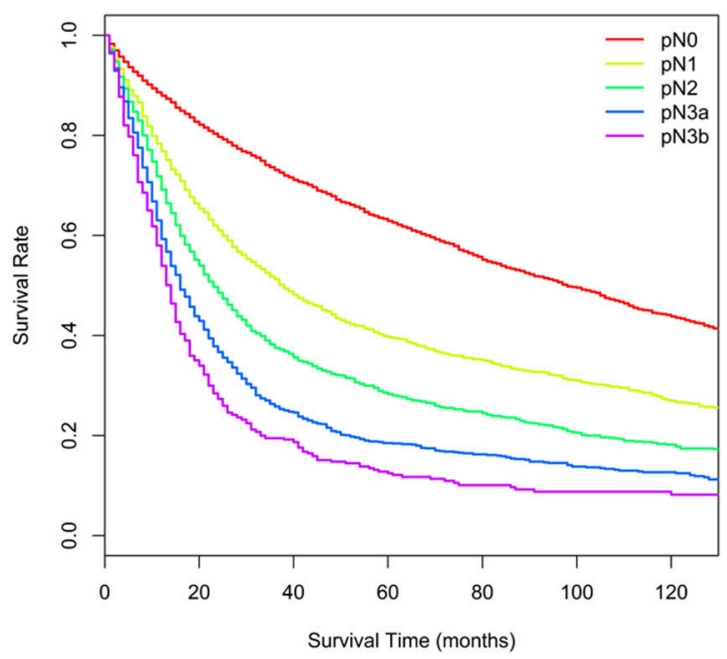

C

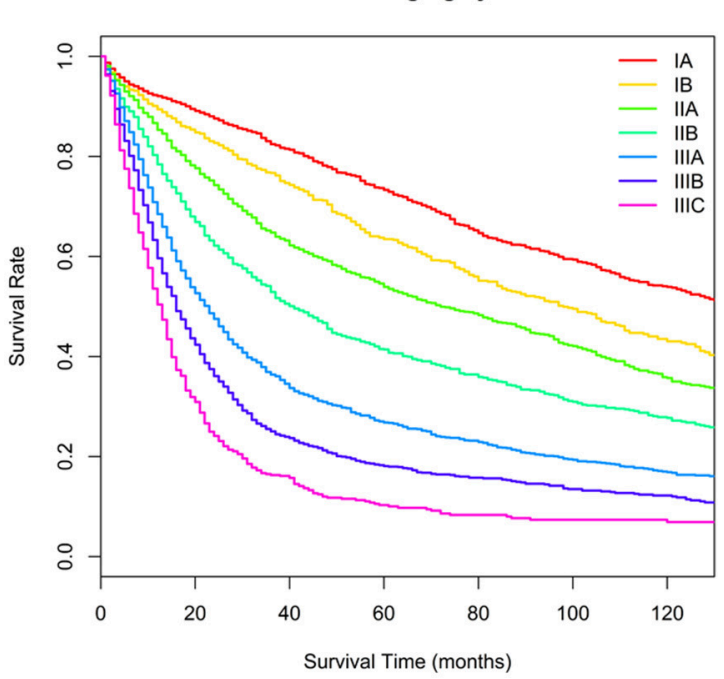

B

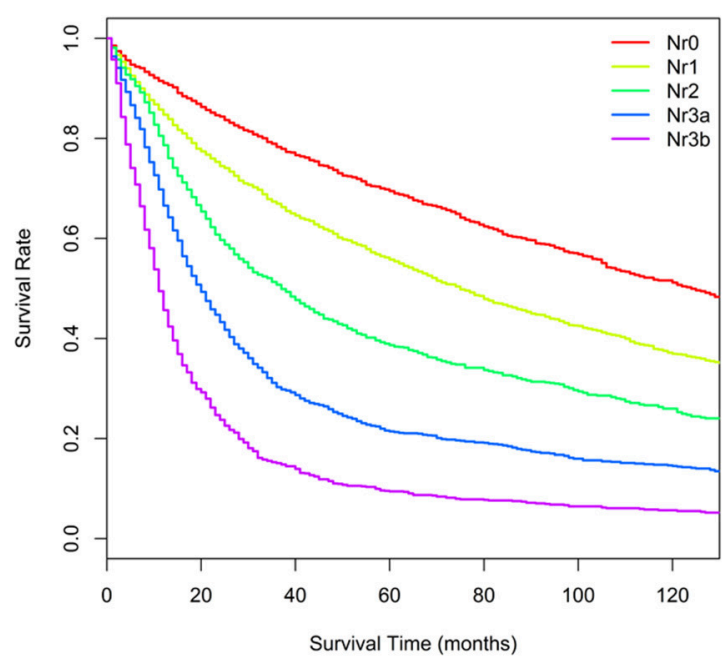

D

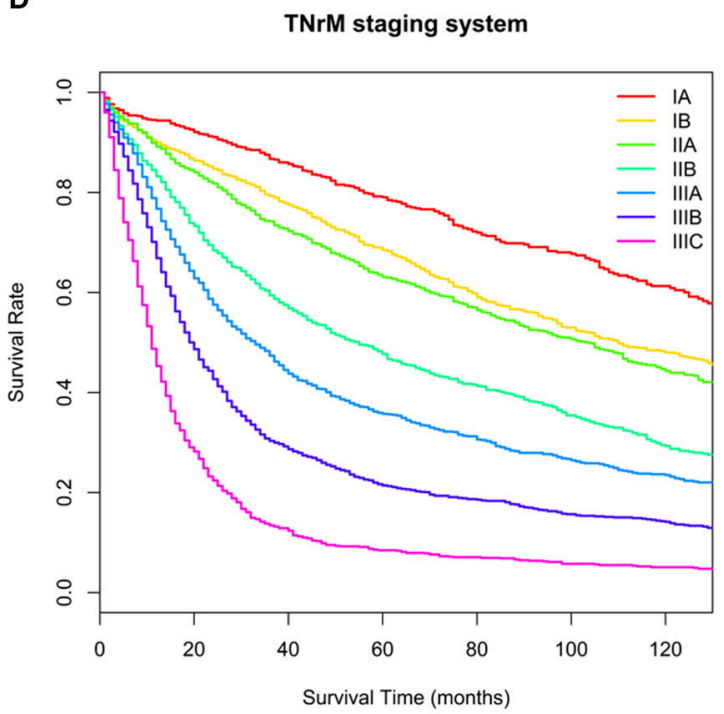

FIGURE 3 | Overall survival (OS) for the validation set based on different staging systems. (A) pN staging system. Five-year OS for the pN categories (pNO-3b) was 62.9, 40.0, 28.3, 18.5, and 12.5\%, respectively. (B) Nr staging system. Five-year OS for the Nr categories (NrO-3b) was 69.4, 55.8, 38.7, 21.4, and 9.4 \%, respectively. (C) 8th TNM staging system. Five-year OS for the 8th TNM stages (IA-IIIC) was 73.3, 63.5, 53.9, 41.4, 26.8, 18.1, and 10.3\%, respectively. (D) TNrM staging system. Five-year OS for the TNrM stages (IA-IIIC) was 79.0, 68.5, 63.1, 47.7, 35.8, 21.4, and 8.5, respectively.

staging system compared with the 7th TNM staging system was to include $\mathrm{pN} 3 \mathrm{~b}$ into the staging system (12-15). In our study, patients with $\mathrm{pN} 3 \mathrm{a}$ had a significantly better OS than those with pN3b, and the 8th TNM staging system was superior to the 7 th TNM staging system when predicting survival for GC patients with $>15$ eLNs, these results were consistent with previous studies (12-15). However, for all patients (included those with $\leq 15$ eLNs), the AJCC 8th TNM staging system was comparable with the 7th TNM staging system, which suggested that the number of eLNs had an influence on the capacity of AJCC TNM prognosis assessment.

Previous studies suggested that the TNrM staging system may better stratify patients' survival than the AJCC TNM staging system (5-11), which was validated in our study. The TNrM staging system satisfied three ideal models of lymph node staging conditions: (1) similar survival rates within a stage group, (2) decreased survival rates with increasing stages, and (3) difference in survival between different stages (21). The main superiority of the TNrM staging system compared with the AJCC TNM staging system is to minimize the stage migration phenomenon. The node-negative patients with fewer than 10 eLNs may not truly be node-negative but rather understaged $(17,22)$. The stage pN1 can be upstaged to $\mathrm{pN} 2$ or even $\mathrm{pN} 3$ as more lymph nodes are examined. Furthermore, it is impossible to be categorized as $\mathrm{pN} 3 \mathrm{~b}$ if fewer than 16 lymph nodes are examined. On the contrary, the TNrM staging system has been proposed to 
reduce the difference of OS within subgroups with $\leq 15$ and $>15$ eLNs $(5,11)$. Similar results were found in our study: the OS of each $\mathrm{N}$ category ( $\mathrm{pN} 0-3 \mathrm{a}$ ) patients with $>15$ eLNs was significantly better than that of patients with $\leq 15$ eLNs. Conversely, significantly different OS was only found in the $\mathrm{Nr} 0$ and $\mathrm{Nr} 3 \mathrm{~b}$ categories for patients with $>15$ eLNs and $\leq 15$ eLNs.

However, the risk of stage migration will decrease as the number of eLNs increased. It should be questioned whether the TNrM staging system performed better than the AJCC 8th TNM staging system for patients with adequate eLNs. It is clear that if the TNM staging system performed as well as the TNrM staging system, the application value of the TNrM staging system would be limited as most patients in Asian had $>15$ eLNs $(3,23)$ and the rate of patients with $>15$ eLNs was increasing in Western (24) countries. Therefore, we conducted a population-based study to investigate whether the TNrM staging system maintained its prognostic power for patients with adequate eLNs. For patients with adequate eLNs, the TNrM staging system still had a better prognostic performance than the AJCC TNM staging system in terms of homogeneity and discriminatory ability. Besides the mechanism stage migration, these results may be related to the fact that the number of lymph nodes itself correlates with survival. A randomized controlled trial suggested that an increase in the number of lymph nodes did not correlate with a change in the percent of node-positive patients or the mean number of metastatic lymph nodes (25). Parsons et al. further confirmed that patients with a high number of eLNs were only slightly more likely to be node-positive, while these patients experienced significantly better OS compared with those with fewer eLNs (16). Several studies have hypothesized that the number of lymph nodes might reflect the tumor-host relationship, and a higher number of lymph nodes dissected may simply reflect a host lymphocytic reaction to the tumor, which is associated with long survival (26-28). However, it is impossible for us to answer where the risk of being understaged ends and where a true benefit of number of eLNs begins.

To the best of our knowledge, this was the first study to directly modify the AJCC 8th TNM staging system based on the $\mathrm{Nr}$ when predicting survival for GC patients using a large database with long-term follow-up. Compared with previous research (5$11)$, there were two main modifications in this study. Firstly, node-negative GC patients were classified into $\mathrm{Nr} 0$ and $\mathrm{Nr} 1$ based on the number of eLNs. Further, we proved the validity of this classification. Secondly, the TNrM staging system could be improved with modifications of staging subgroups. However, in order not to further complicate an already complex staging model, the TNrM staging system was established by combining the same pT and pM with the $\mathrm{Nr}$ instead of the pN. Still, several limitations should be noted in this study. First, all patients were selected from the SEER database, which may restrict it to other populations. The number of eLNs for gastric cancer patients in Asia was more than that in Western countries. However, our study verified that the TNrM staging system performed better than the TNM staging system even for patients with adequate eLNs. Thus, we believe similar results will be found in Asian populations. On the other hand, these cutoff points may not be optimal for the other cohorts of patients, and modification of the LNR intervals may be needed. Secondly, patients without negative lymph nodes may have a risk of upstaged because the $\mathrm{Nr}$ stage will be the same $(\mathrm{Nr} 3 \mathrm{~b})$, which will not be influenced by the number of eLNs. However, we believed patients without negative lymph nodes have a higher risk testing positive for residual lymph nodes, which was associated with worse survival rates. These patients were staged as Nr3b, and this may accurately reflect the risk of residual positive lymph nodes. Thirdly, unlike neoadjuvant radiotherapy, we were unable to determine whether the patients received neoadjuvant chemotherapy that might affect pathological judgment. Given that the standard of neoadjuvant has been concurrent chemoradiotherapy, it is reasonable to assume that patients received combined neoadjuvant chemoradiotherapy. We believe that few patients who received only chemotherapy were included in our study. Despite these limitations, the present study was significant because the TNrM staging system resulted in more GC patients being accurately and discriminatively staged without overcomplicating the TNM staging system.

\section{CONCLUSIONS}

The TNrM staging system predicted survival more accurately and discriminatively than the AJCC 8th TNM staging system for GC patients regardless of the number of eLNs, which should be taken into account as a supplemental staging system when predicting survival for GC patients, especially for those with limited eLNs. However, this result should be validated in other populations.

\section{DATA AVAILABILITY}

Publicly available datasets were analyzed in this study. This data can be found here: https://seer.cancer.gov/.

\section{ETHICS STATEMENT}

All patients in this study were collected from the SEER database, and we received permission for using the data.

\section{AUTHOR CONTRIBUTIONS}

Z-LY, J-HL, and C-XW designed this study. Z-LY, M-HZ, X-JH, and Q-WL performed the search and collected data. C-XW rechecked data. Z-LY and J-HL performed analysis. Z-LY wrote the manuscript. All authors reviewed the manuscript.

\section{ACKNOWLEDGMENTS}

We would like to thank the SEER database for the collection of clinical data.

\section{SUPPLEMENTARY MATERIAL}

The Supplementary Material for this article can be found online at: https://www.frontiersin.org/articles/10.3389/fonc. 2019.00329/full\#supplementary-material 


\section{REFERENCES}

1. Fitzmaurice C, Dicker D, Pain A, Hamavid H, Moradi-Lakeh M, MacIntyre MF, et al. The Global Burden of Cancer 2013. JAMA Oncol. (2015) 1:505-27. doi: 10.1001/jamaoncol.2015.0735

2. Edge SB, Compton CC. The American Joint Committee on Cancer: the 7th edition of the AJCC cancer staging manual and the future of TNM. Ann Surg Oncol. (2010) 17:1471-4. doi: 10.1245/s10434-010-0985-4

3. Sano T, Coit DG, Kim HH, Roviello F, Kassab P, Wittekind C, et al. Proposal of a new stage grouping of gastric cancer for TNM classification: International Gastric Cancer Association staging project. Gastric Cancer. (2017) 20:217-25. doi: 10.1007/s10120-016-0601-9

4. Okusa T, Nakane Y, Boku T, Takada H, Yamamura M, Hioki K, et al. Quantitative analysis of nodal involvement with respect to survival rate after curative gastrectomy for carcinoma. Surg Gynecol Obstet. (1990) 170:488-94.

5. Wang J, Dang P, Raut CP, Pandalai PK, Maduekwe UN, Rattner DW, et al. Comparison of a lymph node ratio-based staging system with the 7th AJCC system for gastric cancer: analysis of 18,043 patients from the SEER database. Ann Surg. (2012) 255:478-85. doi: 10.1097/SLA.0b013e31824857e2

6. Kutlu OC, Watchell M, Dissanaike S. Metastatic lymph node ratio successfully predicts prognosis in western gastric cancer patients. Surg Oncol. (2015) 24:84-88. doi: 10.1016/j.suronc.2015.03.001

7. Lee YC, Yang PJ, Zhong Y, Clancy TE, Lin MT, Wang J. Lymph node ratiobased staging system outperforms the seventh AJCC system for gastric cancer: validation analysis with National Taiwan university hospital cancer registry. Am J Clin Oncol. (2017) 40:35-41. doi: 10.1097/COC.0000000000000110

8. Zhao LY, Li CC, Jia LY, Chen XL, Zhang WH, Chen XZ, et al. Superiority of lymph node ratio-based staging system for prognostic prediction in 2575 patients with gastric cancer: validation analysis in a large single center. Oncotarget. (2016) 7:51069-81. doi: 10.18632/oncotarget.9714

9. Kong SH, Lee HJ, Ahn HS, Kim JW, Kim WH, Lee KU, et al. Stage migration effect on survival in gastric cancer surgery with extended lymphadenectomy: the reappraisal of positive lymph node ratio as a proper N-staging. Ann Surg. (2012) 255:50-8. doi: 10.1097/SLA.0b013e31821d4d75

10. Lee JH, Kang JW, Nam BH, Cho GS, Hyung WJ, Kim MC, et al. Correlation between lymph node count and survival and a reappraisal of lymph node ratio as a predictor of survival in gastric cancer: A multi-institutional cohort study. Eur J Surg Oncol. (2017) 43:432-9. doi: 10.1016/j.ejso.2016.09.007

11. Marchet A, Mocellin S, Ambrosi A, Morgagni P, Garcea D, Marrelli D, et al. The ratio between metastatic and examined lymph nodes ( $\mathrm{N}$ ratio) is an independent prognostic factor in gastric cancer regardless of the type of lymphadenectomy: results from an Italian multicentric study in 1853 patients. Ann Surg. (2007) 245:543-52. doi: 10.1097/01.sla.0000250423.43436.e1

12. In H, Solsky I, Palis B, Langdon-Embry M, Ajani J, Sano T. Validation of the 8th edition of the AJCC TNM staging system for gastric cancer using the National Cancer Database. Ann Surg Oncol. (2017) 24:3683-91. doi: 10.1245/s10434-017-6078-x

13. Kim SG, Seo HS, Lee HH, Song KY, Park CH. Comparison of the Differences in Survival Rates between the 7th and 8th Editions of the AJCC TNM Staging System for Gastric Adenocarcinoma: a Single-Institution Study of 5,507 Patients in Korea. J Gastric Cancer. (2017) 17:212-9. doi: 10.5230/jgc.2017.17.e23

14. He X, Wu W, Lin Z, Ding Y, Si J, Sun LM. Validation of the American Joint Committee on Cancer (AJCC) 8th edition stage system for gastric cancer patients: a population-based analysis. Gastric Cancer. (2017) 21:391-400. doi: 10.1007/s10120-017-0770-1

15. Ji X, Bu ZD, Yan Y, Li ZY, Wu AW, Zhang LH, et al. The 8th edition of the American Joint Committee on Cancer tumor-node-metastasis staging system for gastric cancer is superior to the 7 th edition: results from a Chinese mono-institutional study of 1663 patients. Gastric Cancer. (2017) 21:643-52. doi: 10.1007/s10120-017-0779-5

16. Parsons HM, Tuttle TM, Kuntz KM, Begun JW, McGovern PM, Virnig BA. Association between lymph node evaluation for colon cancer and node positivity over the past 20 years. JAMA. (2011) 306:1089-97. doi: 10.1001/jama.2011.1285

17. Yang ZL, Zhu MH, Shi Q, Lu FM, Wang CX. Prognostic value of the number of lymph nodes examined in patients with node-negative gastric cancer. $J$ Gastrointest Surg. (2018) 23:460-7. doi: 10.1007/s11605-018-3947-y

18. Camp RL, Dolled-Filhart M, Rimm DL. X-tile: a new bio-informatics tool for biomarker assessment and outcome-based cut-point optimization. Clin Cancer Res. (2004) 10:7252-9. doi: 10.1158/1078-0432.CCR-04-0713

19. Chaurasia A, Harel O. Using AIC in multiple linear regression framework with multiply imputed data. Health Serv Outcomes Res Methodol. (2012) 12:219-33. doi: 10.1007/s10742-012-0088-8

20. Ajani JA. National Comprehensive Cancer Network Gastric Cancer Guidelines. National Comprehensive Cancer Network 2018: Version 1.2018.

21. Ueno S, Tanabe G, Sako K, Hiwaki T, Hokotate H, Fukukura Y, et al. Discrimination value of the new western prognostic system (CLIP score) for hepatocellular carcinoma in 662 Japanese patients. Cancer of the Liver Italian Program. Hepatology. (2001) 34:529-34. doi: 10.1053/jhep.2001.27219

22. Smith DD, Schwarz RR, Schwarz RE. Impact of total lymph node count on staging and survival after gastrectomy for gastric cancer: data from a large US-population database. J Clin Oncol. (2005) 23:7114-24. doi: 10.1200/JCO.2005.14.621

23. Ahn HS, Lee HJ, Hahn S, Kim WH, Lee KU, Sano T, et al. Evaluation of the seventh American Joint Committee on Cancer/International Union Against Cancer Classification of gastric adenocarcinoma in comparison with the sixth classification. Cancer. (2010) 116:5592-8. doi: 10.1002/cncr.25550

24. Arsoniadis EG, Marmor S, Diep GK, Hui JYC, Jensen EH, Tuttle TM, et al. Survival rates for patients with resected gastric adenocarcinoma finally have increased in the United States. Ann Surg Oncol. (2017) 24:3361-7. doi: 10.1245/s10434-017-5992-2

25. Wu CW, Hsiung CA, Lo SS, Hsieh MC, Chen JH, Li AF, et al. Nodal dissection for patients with gastric cancer: a randomised controlled trial. Lancet Oncol. (2006) 7:309-15. doi: 10.1016/S1470-2045(06)70623-4

26. Willaert W, Mareel M, Van De Putte D, Van Nieuwenhove Y, Pattyn P, Ceelen W. Lymphatic spread, nodal count and the extent of lymphadenectomy in cancer of the colon. Cancer Treat Rev. (2014) 40:405-13. doi: 10.1016/j.ctrv.2013.09.013

27. George S, Primrose J, Talbot R, Smith J, Mullee M, Bailey D, et al. Will Rogers revisited: prospective observational study of survival of 3592 patients with colorectal cancer according to number of nodes examined by pathologists. $\mathrm{Br}$ J Cancer. (2006) 95:841-7. doi: 10.1038/sj.bjc.6603352

28. Pages F, Galon J, Fridman WH. The essential role of the in situ immune reaction in human colorectal cancer. J Leukoc Biol. (2008) 84:981-7. doi: 10.1189/jlb.1107773

Conflict of Interest Statement: The authors declare that the research was conducted in the absence of any commercial or financial relationships that could be construed as a potential conflict of interest.

Copyright (c) 2019 Yang, Zhu, Han, Liu, Long and Wang. This is an open-access article distributed under the terms of the Creative Commons Attribution License (CC $B Y)$. The use, distribution or reproduction in other forums is permitted, provided the original author(s) and the copyright owner(s) are credited and that the original publication in this journal is cited, in accordance with accepted academic practice. No use, distribution or reproduction is permitted which does not comply with these terms. 